\title{
Differences between carotene and retinol
}

By RichaRd Peto, Imperial Cancer Research Fund Studies Unit, Nuffield Department of Clinical Medicine, Radcliffe Infirmary, Oxford $O X_{2} 6 H E$

Among most people in developed countries, blood retinol levels do not depend strongly on the amounts of retinol derived from vitamin A (i.e. retinyl esters) or provitamin A (i.e. certain carotenoids) in the diet. So, varying these dietary intakes is unlikely, except possibly for people who are at least marginally deficient in vitamin $A$, to have any material effect on cancer via modifying blood retinol levels. If dietary vitamin $A$ intake is at all relevant to cancer prevention in developed countries it is more likely to act via modification of blood carotene, rather than blood retinol, levels. (There are various theoretical ways that carotenoids might be protective, although none are of demonstrated relevance; these include free-radical trapping, quenching of excited species, or acting as a source of topical provitamin $A$ in the tissues that are to be protected.) Conversely, if circulating retinol (or other retinoids) is directly protective against cancer, then although any factors that importantly determine blood retinol levels may be important modifiers of cancer onset rates, these factors are not yet understood. However, for most people in developed countries these factors do not appear to include vitamin $A$ intake.

A review article overlapping considerably with the content of my lecture has recently been published (Peto et al. 1981).

\section{REFERENCE}

Peto, R., Doll, R., Buckley, J. D. \& Sporn, M. B. (1981). Nature, Lond. 290, 201. 